\title{
A Method to Determine the Flexural Rigidity of the Main Dipole for the Large Hadron Collider
}

\author{
M. La China, G. Cavallari, P. Ferracin, J. Garcia Perez, E. Todesco, and W. Scandale
}

\begin{abstract}
The Large Hadron Collider (LHC) superconducting dipole cold mass is a cylindrical structure $15 \mathrm{~m}$ long, made of a shrinking cylinder which contains iron laminations and collared coils. This structure, weighing about 28 ton is horizontally bent by 5 mrad. Its shape should be preserved from the assembly phase to the operational condition at cryogenic temperature. Hence an accurate comprehension of the mechanical behavior of the cold mass is required. In particular the flexural rigidity in horizontal and vertical directions represents one of the foremost properties to be aware of. To determine the flexural rigidity, deformations of the cold mass induced by the self weight have been measured and compared with the predictions of an analytical structural model. A particular care has been taken in reducing the experimental error by an appropriate fitting procedure.
\end{abstract}

Index Terms-Optical interferometry, structural engineering superconducting magnet.

\section{INTRODUCTION}

$\mathbf{T}$ 7 HE LHC main dipole is a twin aperture magnet $14.8 \mathrm{~m}$ long, $0.57 \mathrm{~m}$ diameter, weighing 28 ton. The $8.3 \mathrm{~T}$ nominal field is reached at $11.8 \mathrm{kA}$ at the operational temperature of $1.9 \mathrm{~K}$. The magnet cross section is shown in Fig. 1. The superconducting coils, surrounding the two apertures, are constrained in radial and azimuthal directions by austenitic steel collars. The iron yoke surrounding the collar screens the magnetic field outside the magnet and enhances it by around $18 \%$. The described components, kept in a $1.9 \mathrm{~K}$ helium bath and packed in by a shrinking cylinder, form the so-called "cold mass."

The foremost cold mass structural characteristic is the internal discontinuity due to the absence of any weld between the various components. In the radial direction, in fact, the superconducting cables, the steel collar and the iron yoke are simply packed together by the shrinking cylinder. Moreover the collar and the yoke, being made of $3 \mathrm{~mm}$ and $6 \mathrm{~mm}$ thick laminations, are kept in the longitudinal direction by steel rods. As a consequence, over the 14.8 meters dipole length, the only continuous structural elements are the cables, the shrinking cylinder and the rods.

Because of the internal structure complexity, it is impossible to deduce the structural properties of the cold mass in an analytical way. Tests and models have already been worked out [1], [2] but some not negligible discrepancies in the results show the need to keep on studying this feature. This paper describes ex-

Manuscript received August 6, 2002.

The authors were with the European Organization for Nuclear Research CERN, CH-1211 Geneva 23, Switzerland at the time of submittal (e-mail marco.la.china@cern.ch).

P. Ferracin is now with the Lawrence Berkeley National Laboratory (e-mail: pferracin@lbl.gov).

Digital Object Identifier 10.1109/TASC.2003.812655

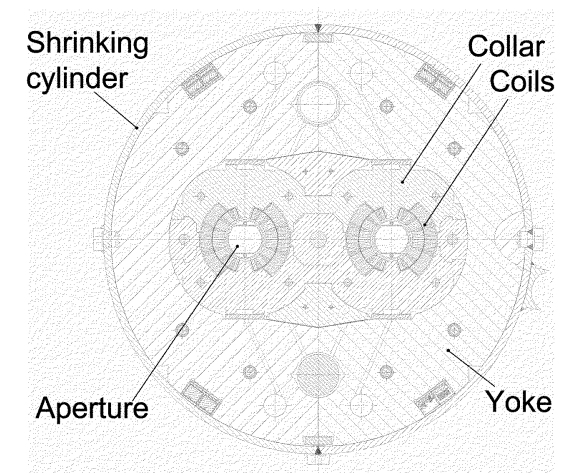

Fig. 1. Cross section of the cold mass of the main LHC dipole.

perimental tests and an analytical model used to determine the flexural rigidity of the LHC superconducting dipole.

In Section II we describe the cold mass measuring set up; the analytical model used to interpret experimental data, the evaluation of the flexural rigidity and the error estimates are given in Section III. Conclusions are drawn in Section IV.

\section{EXPERIMENTAL DATA}

In our set up, the cold mass is placed on two sets of wheelshaped supports located approximately at the quarter points as shown in Fig. 2. With respect to the reference system shown in Fig. 2 the cold mass has been loaded in $Z$ and $Y$ directions and the flexion in the corresponding $Z X$ and $Y X$ planes has been measured. The large cold mass dimensions compared to the small deformations induced by the load require a measuring device based on interferometric technologies. The Leica ${ }^{\circledR}$ LTD500 laser tracker system, normally employed to provide metrological informations during magnet assembly [3], has been used without essential modifications. The relevant features of this instrument are summarized in Table I.

The laser reflector is installed on a cylindrical mole traveling inside one of the two apertures [4]. Tracking its position in the three dimensions provides information on the aperture shape in around 100 points along the magnet axis with an accuracy of 5 p.p.m. $(5 \mu \mathrm{m} / \mathrm{m})$ at $95 \%$ confidence level.

In the $Z$-axis direction the load is provided by the cold mass self-weight acting uniformly along the magnet length. Such a static load induces maximum deflections in the $X Z$ plane of about $1 \mathrm{~mm}$. Indeed the $\pm 1 \mathrm{~mm}$ fabrication tolerance is of the same order of magnitude of the deflection, and therefore cannot be neglected. To cancel this effect, the magnet has been turned by 180 degrees around the $X$-axis and the vertical deflection has been measured again. 


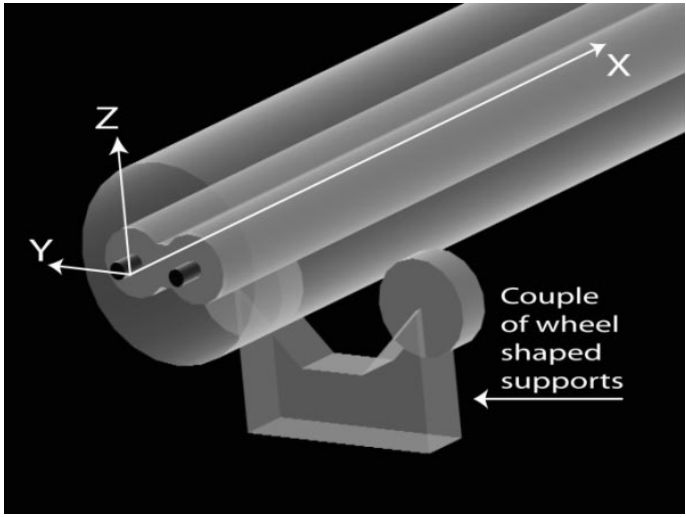

Fig. 2. Cold mass supports during test. This kind of support permits the rotation of the magnet around its longitudinal axis. This feature has been used to perform measurements of the cold mass axis in different positions with respect to the gravitational load.

TABLE I

LASER TRACKER MAIN FEATURES

\begin{tabular}{ll}
\hline \multicolumn{2}{c}{ LEICA LTD500 ACCURACY @2 $2 \sigma$} \\
\hline Angle resolution & $0.14 "$ \\
Distance resolution & $1.26 \mu \mathrm{m}$ \\
Reproducibility of a co-ordinate & $\pm 5 \mathrm{ppm}(\mu \mathrm{m} / \mathrm{m})$ \\
Absolute accuracy of a co-ordinate & \\
& \\
- Cor non-moving target (static) & $\pm 10 \mathrm{ppm}(\mu \mathrm{m} / \mathrm{m})$ \\
- Cor moving target (dynamic) & $\pm 20-40 \mathrm{ppm}(\mu \mathrm{m} / \mathrm{m})$ \\
\hline
\end{tabular}

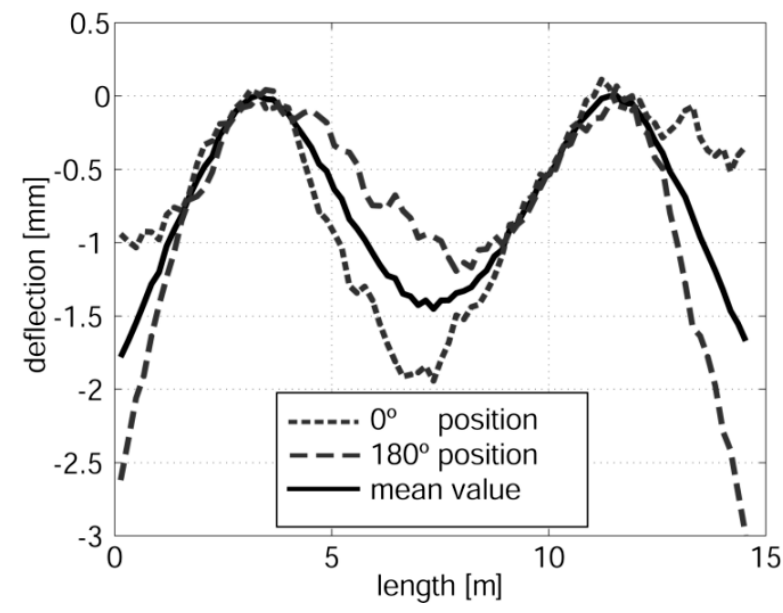

Fig. 3. Magnet deflections along the axis in the vertical plane and their average. The effect of the manufacturing misalignments is clearly visible in both the positions whereas is absent in their average.

In Fig. 3 the acquired data are referred to an absolute reference system. The dotted lines represent the axis deflections in the vertical plane for two opposite up-down cold mass positions. The average of those two measurements can be assumed to be the deformed shape of a theoretically straight cold mass, thus unaffected by the manufacturing misalignments. Indeed, the large difference in the shape between the $0^{\circ}$ and $180^{\circ}$ positions (see Fig. 3) confirms that fabrication tolerances are not negligible. This effect is absent when the average shape is considered.

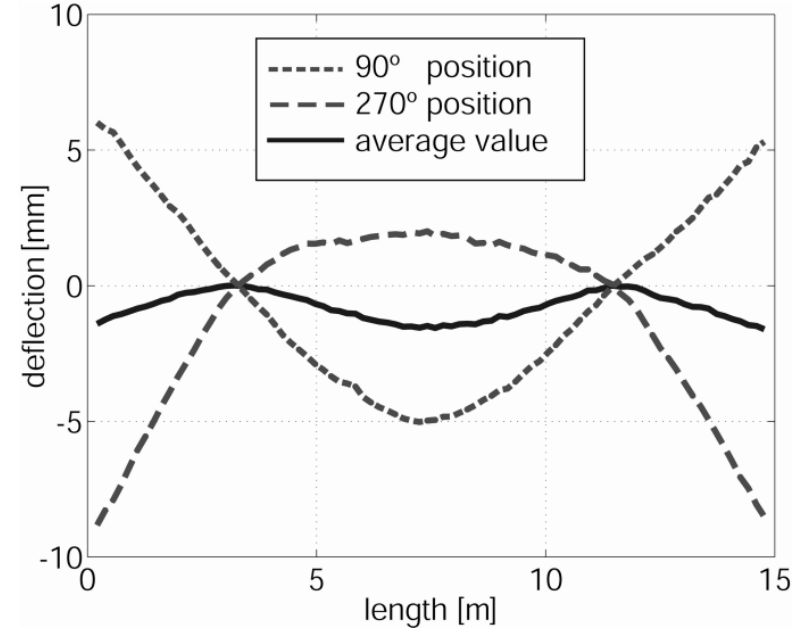

Fig. 4. Magnet deflections along the axis in the horizontal plane and their average. The effect of the horizontal curvature is clearly visible in both the positions whereas is absent in their average.

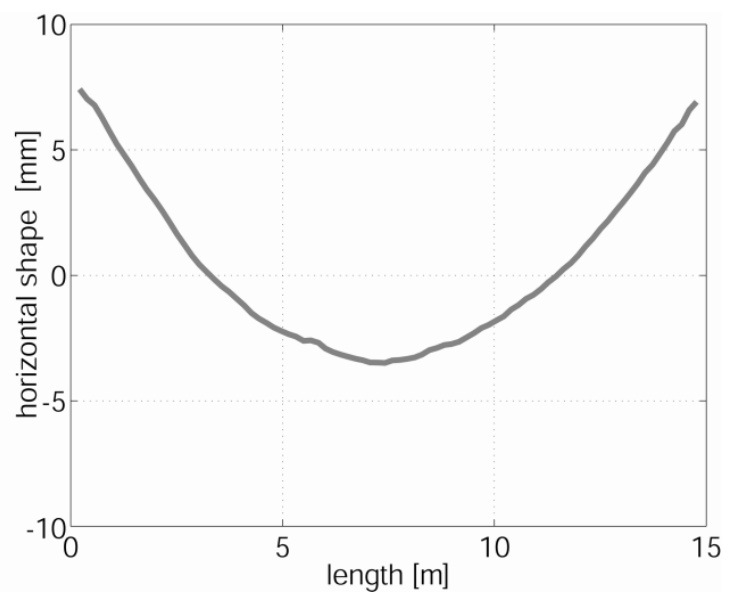

Fig. 5. The axis horizontal curvature of about $5 \mathrm{mrad}$ has been obtained by processing the two opposite measurements performed in the $90^{\circ}$ and in the $270^{\circ}$ positions.

To measure the magnet deflection in the $X Y$ plane induced by a load applied along the $Y$-axis direction, the self-weight has been used again. The procedure has been exactly the same as previously described except for the initial magnet position that has been turned by 90 degrees around the longitudinal axis to make the gravity load work in the $Y$ direction.

The results of the measurements are shown in Fig. 4. It is clearly visible how the horizontal curvature of about $5 \mathrm{mrad}$ strongly affects the axis deflection in both the positions whereas it does not have any influence on the average shape.

This double measurement allows for a very precise estimation of magnet axis shape. In fact a simple handling of the acquired data provides the magnet shape in the horizontal and vertical plane in a load-free state.

The axis shape in the horizontal plane is obtained dividing by two the difference between the measurements at $90^{\circ}$ and $270^{\circ}$. The result, shown in Fig. 5, is an arc of circle with a curvature of about $5 \mathrm{mrad}$ (the magnet curvature is designed to provide the maximum aperture clearance to the circulating beams). 


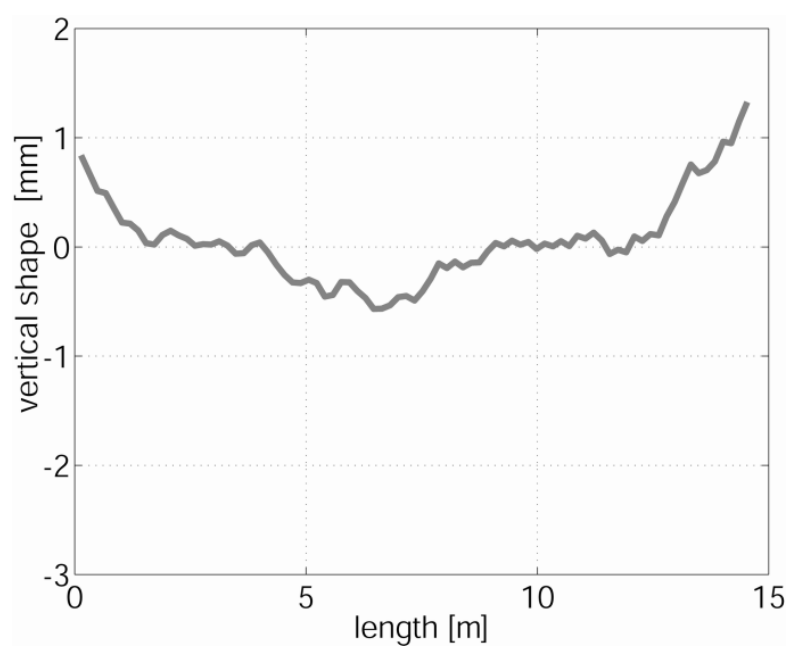

Fig. 6. The manufacturing errors on the axis straightness in the vertical plane are clearly visible in this plot. This axis plot, in load-free condition, has been obtained by processing the data taken in the $0^{\circ}$ and $180^{\circ}$ positions.

The same analysis is carried out in Fig. 6 for the vertical plane $\left(0^{\circ}\right.$ and $180^{\circ}$ positions). This gives the shape of the dipole axis in the vertical plane in a load-free condition that has to be within $\pm 1 \mathrm{~mm}$ tolerances. The large deviations (around $1 \mathrm{~mm}$ ) from the theoretical straight line would have detrimentally affected the accuracy of the final result.

\section{EVALUATION OF FLEXURAL RIGIDITY}

The large length-over-diameter ratio allows treating the cold mass as a thin beam; moreover, the curvature in the horizontal plane of about $5 \mathrm{mrad}$ can be neglected. Studies on the magnet elasticity [5] ensure the absence of any plastic behavior in the range of the observed deformations (about $1 \mathrm{~mm}$ ). Under these assumptions, the axis deflection is simply due to the flexural moments arising on the cross section (pure bending theory) and it can be analytically described by a 3-equations system representing the "deflection function" $Z$. This function, schematized in Fig. 7, has 7 main parameters:

- Longitudinal coordinate $X$

- Flexural rigidity EI

- Support A position $X a$

- Support B position $X b$

- Support height difference $h$

- Linear density $\rho$

- Magnet length $L$.

The flexural rigidity EI is to be evaluated by analyzing experimental data in this analytical framework. The accuracy concerning this estimate is characterized by the overall precision of the remaining 6 input parameters. For this reason a sensitivity analysis has been worked out [2] on each parameter. A large effect of the support horizontal position on the flexural rigidity estimate has been observed. This sensitivity cannot be ignored since, in the analytical model, the supports are represented by point-like constraints whereas in the test they are represented by two sets of $10 \mathrm{~cm}$ wide wheels. Consequently the equivalent contact point could be anywhere within that $10 \mathrm{~cm}$ and this uncertainty $( \pm 5 \mathrm{~cm})$ can strongly affect the accuracy of the computed EI value. To overcome this lack of precision not only EI

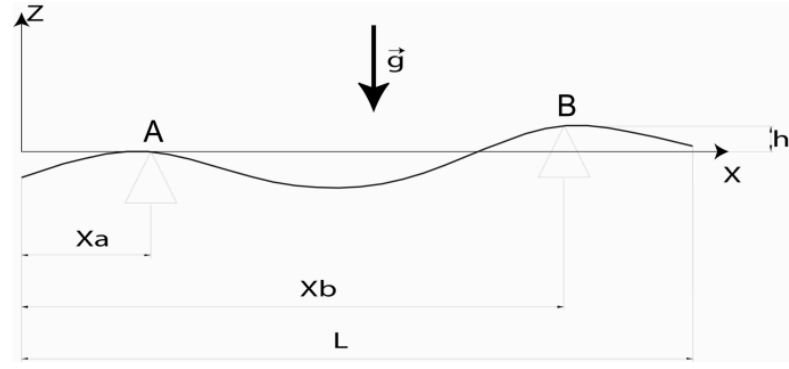

Fig. 7. Analytical function of the axis deflection.

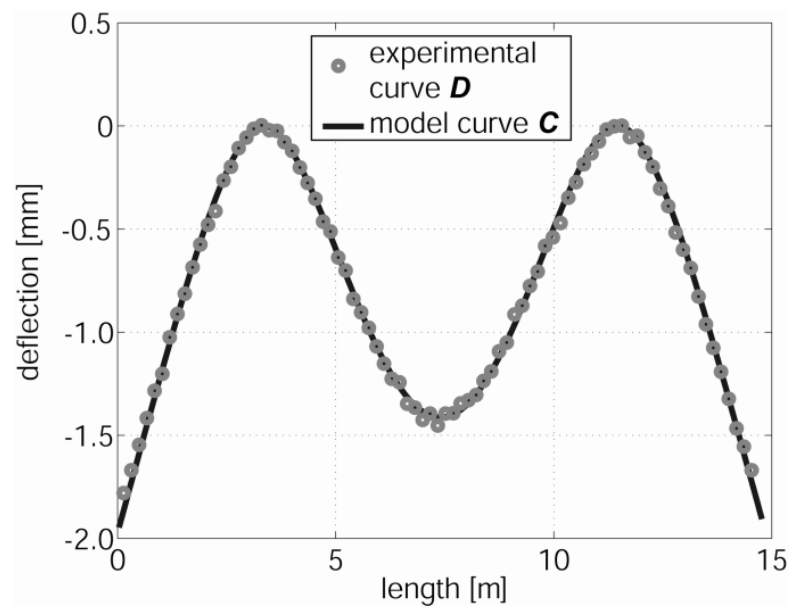

Fig. 8. Vertical deflection of dipole axis along its longitudinal length: experimental data (markers) and best fit of analytical model (solid line).

but also the support positions has been considered as main unknowns.

To fit the model on the data a least square method has been chosen. The previously mentioned fitting variables are represented by EI, $X a$ and $X b$ whereas the remaining model parameters are taken as constant input. The measured difference $h$ between the support heights (see Fig. 7) of about $1 \mathrm{~cm}$ has been neglected. This approximation does not affect the result accuracy. Indeed the distance between the supports is around $8 \mathrm{~m}$, hence the only error introduced is a rotation of the gravity direction of about $1 \mathrm{mrad}$.

In Fig. 8 are shown the axis vertical deflection as measured and as computed by the least square fitting routine. It can be noticed how the match between the analytical and the experimental curve is good in every position along the magnet.

In Fig. 9 are shown the axis deflection in the horizontal plane as measured and as computed by the least square fitting routine. The match between the analytical and the experimental curve is quite good. Small discrepancies can be noticed only near the support placed at 11.5 meters.

The obtained best fit parameters are given in Table II. It can be pointed out that equivalent contact points positions $X a$ and $X b$ outside a range of $5 \mathrm{~cm}$ around center of the real support positions $(3.3 \mathrm{~m}$ and $11.5 \mathrm{~m}$ ) would have been inconsistent. The estimated value of the flexural rigidity is around $160 \mathrm{MPa} \mathrm{m}^{4}$ in both directions. This agrees within $15 \%$ with previous estimates [1], [2].

The sensitivity analysis previously mentioned pointed out the weak influence of the input data precision except for that related 


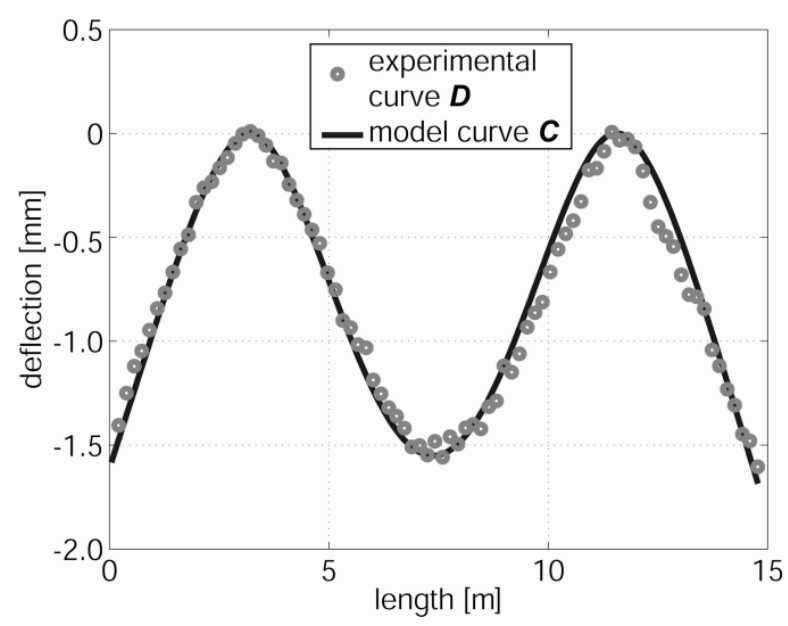

Fig. 9. Horizontal deflection of dipole axis along its longitudinal length: experimental data (markers) and best fit of analytical model (solid line).

TABLE II

BEST Fit OF EXPERIMENTAL DATA: FLEXURAL Rigidity EI AND POSITION OF THE SUPPORT POSTS $X A, X B$

\begin{tabular}{cccc}
\hline & $\mathrm{EI}\left[\mathrm{Mpa} \mathrm{m}^{4}\right]$ & $\mathrm{Xa}[\mathrm{m}]$ & $\mathrm{Xb}[\mathrm{m}]$ \\
\hline Vertical & $159 \pm 4$ & 3.331 & 11.474 \\
Horizontal & $162 \pm 4$ & 3.293 & 11.478 \\
\hline
\end{tabular}

to the support positions. Since the support positions have been included as independent variables in the fitting procedure, the main error source can be considered related to the residual $\boldsymbol{R}_{f}$ between the analytical curve $\boldsymbol{C}$ and the experimental curve $\boldsymbol{D}$.

To estimate the error related to $\boldsymbol{R}_{f}$ a Montecarlo based simulation has been carried out. The result of this simulation is a distribution of EI values centered on the flexural rigidity previously obtained through the fitting procedure. Each EI value has been obtained through a fitting procedure on new curves as done on $\boldsymbol{D}$. Every curve has been generated by a random extraction of points enclosed in a band around the original data $D$. The points in the band follow a normal distribution centered on the experimental data $\boldsymbol{D}$ and with a standard deviation equal to $\boldsymbol{R}_{f}$. The RMS of the EI distribution has been taken as the error on the flexural rigidity @ $1 \sigma$.

The result is $\pm 2 \mathrm{MPa} \mathrm{m}^{4}$ both in vertical and in the horizontal planes.

\section{CONCLUSIONS}

The flexural rigidity of the superconducting dipole for the LHC has been determined through the comparison of an analytical model with data coming from a dedicated test. The test purpose was to measure the magnet axis deflection due to a mechanical static load. The self-weight has been chosen as load and to measure the small deformations a laser tracker has been used. Different load cases have been performed to cancel out the error induced by the manufacturing tolerances. Support positions have been considered as fit parameters (within the expected values) to increase the accuracy of the method. The obtained value of the flexural rigidity is of around $160 \mathrm{MPa} \mathrm{m}^{4}$ in both directions.

\section{REFERENCES}

[1] D. Bozzini, G. Calvino-Palacios, A. Roussellin, B. Skoczen, C. Vuitton, and L. R. Williams, "Bending tests on $10 \mathrm{~m}$ and $15 \mathrm{~m}$ LHC dipole cold masses," CERN LHC-CRI Technical Note 98-16.

[2] M. Bajko, R. Chamizo, and A. Pardons, "Influence of geometrical parameters on the flexural rigidity of the LHC dipole cold mass assembly," in 17th International Conference on Magnet Technology (MT17), Geneva, Switzerland, Sept. 24-28, 2001.

[3] M. Bajko, A. Pardons, and F. Savary, "Metrology of the LHC dipole cold masses," in 17th International Conference on Magnet Technology (MT17), Geneva, Switzerland, Sept. 24-28, 2001.

[4] S. Aznar et al., "A novel device for the measurement of the mechanical and magnetic axes of the superconducting magnet assemblies for accelerators," in 17th International Conference on Magnet Technology (MT17), Geneva, Switzerland, Sept. 24-28, 2001.

[5] M. La China, J. Garcia Perez, G. Gubello, W. Scandale, and E. Todesco, "Geometrical position of the LHC main dipole inside the cryostat," in 8th European Particle Accelerator Conference (EPAC2002), Paris, France, June 3-7, 2002. 